\title{
Nanoscale mapping of plasmons, photons, and excitons
}

\section{Mathieu Kociak and Javier García de Abajo}

\begin{abstract}
The possibility has recently been reported of using spatially resolved electron energy loss spectroscopy and cathodoluminescence in scanning (transmission) electron microscopes to probe optical excitations - plasmons, photons, excitons - at a scale that could not have been considered only a few years ago. This allows these excitations to be studied at the relevant scale for the characterization of novel materials with potential applications in nanophotonics and nanoplasmonics. This review aims at describing the state-of-the art experimental and theoretical techniques of this emerging field and its major uses and applications.
\end{abstract}

\section{Introduction}

The optical properties of nanoscale objects drastically change at the nanometer scale as a function of their size, morphology, and environment. Indeed, when the structure of a material exhibits variations on the order of or below the typical wavelength of light, new photonic or plasmonic modes emerge. When it exhibits variations of the order of the excitonic Bohr radius - a few nanometers, new excitonic states develop. Such considerations would have probably remained as research curiosities if the growing availability of new materials, such as photonic bandgap crystals, metallic nanoparticles, or quantum confined systems (quantum wells, quantum dots), had not triggered the need for new tools and new concepts for the exploration and understanding of this fascinating new realm.

Getting spectroscopic information at the nanoparticle scale, or even better, within individual nanoparticles or unit cells of an artificial material, is not simple because the required spatial resolution is one or two orders of magnitude smaller than the equivalent wavelength in a vacuum or in a medium. This information, however, is crucial in many applications (e.g., biosensors based upon plasmons or photodetectors relying on excitons), but using purely optical microscopy is not usually adequate at these scales. An alternative is to use fast electrons beams, taking advantage of the fact that electron energy-loss spectroscopy (EELS) and cathodoluminescence (CL) can be regarded as the nanometer analog of extinction and scattering/ photoluminescence, respectively. These two techniques have for a long time faced similar problems: obtaining, with appropriate spatial and spectral resolutions and signal-to-noise ratio, access to the relevant energy range (IR-visible-UV). After a series of pioneering work both in $\mathrm{EELS}^{1-3}$ and CL, ${ }^{4-7}$ these problems have been solved in past years ${ }^{4,8,9}$ for surface plasmons and more recently for quantum emitters. ${ }^{10}$

The present article is aimed at discussing some of the latest results and applications in mapping and understanding these excitations at the nanometer scale. The interested reader can refer to Reference 11 for a deeper understanding of the theoretical background, Reference 12 for a deeper understanding of technical issues, and References 12 and 13 for information on other spatially resolved electron spectroscopy findings and applications.

\section{Experimental and simulation techniques}

Figure 1 presents a simple system for performing both EELS and CL experiments that can be realized in a scanning (transmission) electron microscope, S(T)EM. A fast electron beam (1 keV to $30 \mathrm{keV}$ for SEM, $40 \mathrm{keV}$ to $300 \mathrm{keV}$ for a STEM) ranging from a few nanometers to tenths of nanometers in diameter is scanned over the sample of interest. At each position of the beam, basically two different kinds of signals of interest can be detected. The first kind enables the recording of images of the sample: the high angle annular dark-field (HAADF) signal 


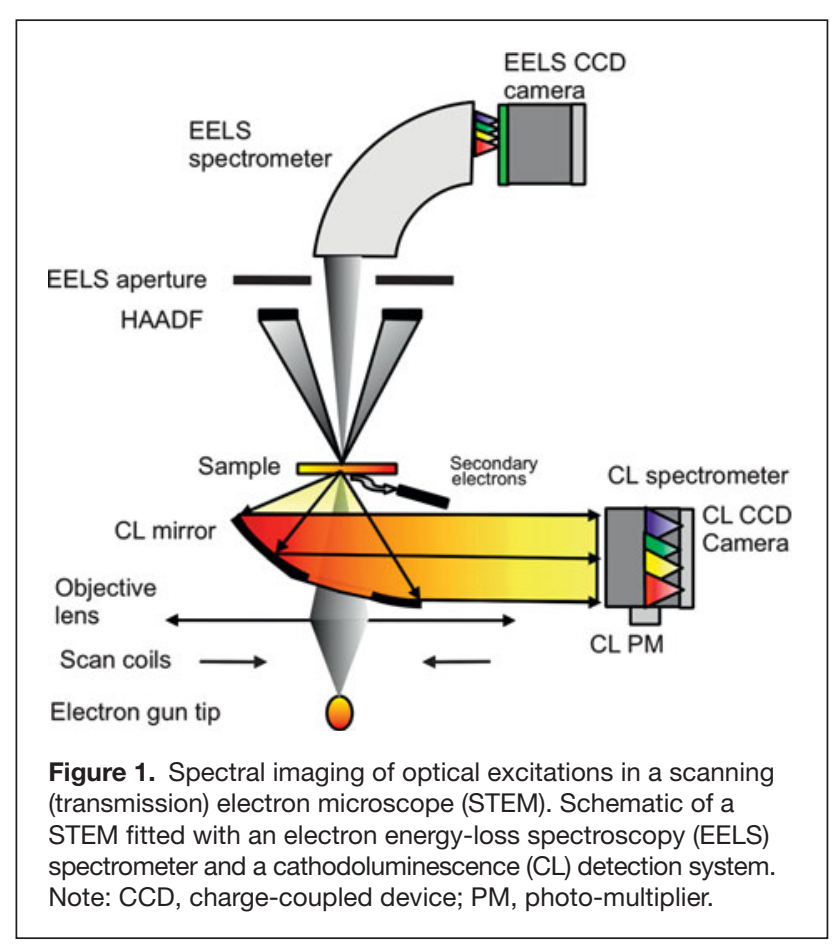

is roughly proportional to the projected mass under the beam, and the secondary or backscattered electron signals are related to the surface structure. The second kind enables the formation of spectral images (i.e., images with a spectrum in each pixel). ${ }^{14}$ The EELS signal is related to the energy lost by an electron through its interaction with a given sample. If the energy lost is in the near infrared/visible/ultraviolet (IR-vis-UV) range, EELS gives useful information on the optical properties of the sample, although the main use of EELS in a STEM has been the study of nanoscale chemistry through the analysis of higher energy ranges (see References 12 and 15). When the energy absorbed from the electron is re-emitted in the form of light, a CL spectrum can be measured and gives yet another set of useful information on the optical properties. It is worth noting that every image, either $2 \mathrm{D}$ or $3 \mathrm{D}$, can be usually recorded in parallel, allowing a direct, nanometer scale correlation between the structure and the spectroscopic signal, which is a decisive advantage of these techniques for precisely comparing the structure/optical property relations. While less widespread, another related setup where filtered images rather than spectra are acquired allows one to perform EELS spectral imaging. ${ }^{16,17}$ Such a setup has been shown to have high performance for large images ${ }^{12,16}$ (see Reference 18 for a relevant review).

Figure 2a shows the capabilities of the spectral imaging EELS technique for mapping plasmons. (Figure 3a-b gives similar results in the case of CL on a gold nanoantenna. ${ }^{19}$ ) In this example, a spectral image has been taken on a silver prism, the edge length of which is $70 \mathrm{~nm},{ }^{8}$ as shown on the HAADF image. Examples of EELS spectra taken at positions A, B, and C show how well-separated resonances, spanning the near-infrared to ultraviolet regime, can be identified. As one spectrum is taken at each triangle position, and because each peak is well separated, one can automatically fit each of these peaks and plot the relevant information for each of them (see References 8, 10, and 12 for details). Hence, the energy peak position for the first excitation is plotted, showing an almost constant energy position valuesimilar results are observed for the other peaks. The intensity of these peaks changes dramatically over the triangle, and for each energy position, a different spatial distribution is seen. Such results are well reproduced by simulations using the boundary element method (see next section) of the EELS signal. It seems that EELS (and CL, see Figure 3a-b) provide a good image of a plasmon wave. The interpretation of such maps in these terms is described in the next section. It is worth noting that such a combination of spatial and spectral resolutions and signal to noise ratio could not be achieved with pure photon spectroscopy.

\section{Linking electron and photon spectroscopies}

In this section, we discuss the understanding of the EELS and CL spectra and their spatial distribution in the case where the optical phenomena can be accurately described by solving the Maxwell equations with known boundaries and constituent material dielectric functions.

For a long time, EELS has been considered as a way of measuring the optical properties of bulk materials (dielectric constants) or shown to be highly sensitive to surface plasmons. ${ }^{11}$ In situations characterized by high symmetry, such as spheres, planes, or cylinders, surface EELS signals have been

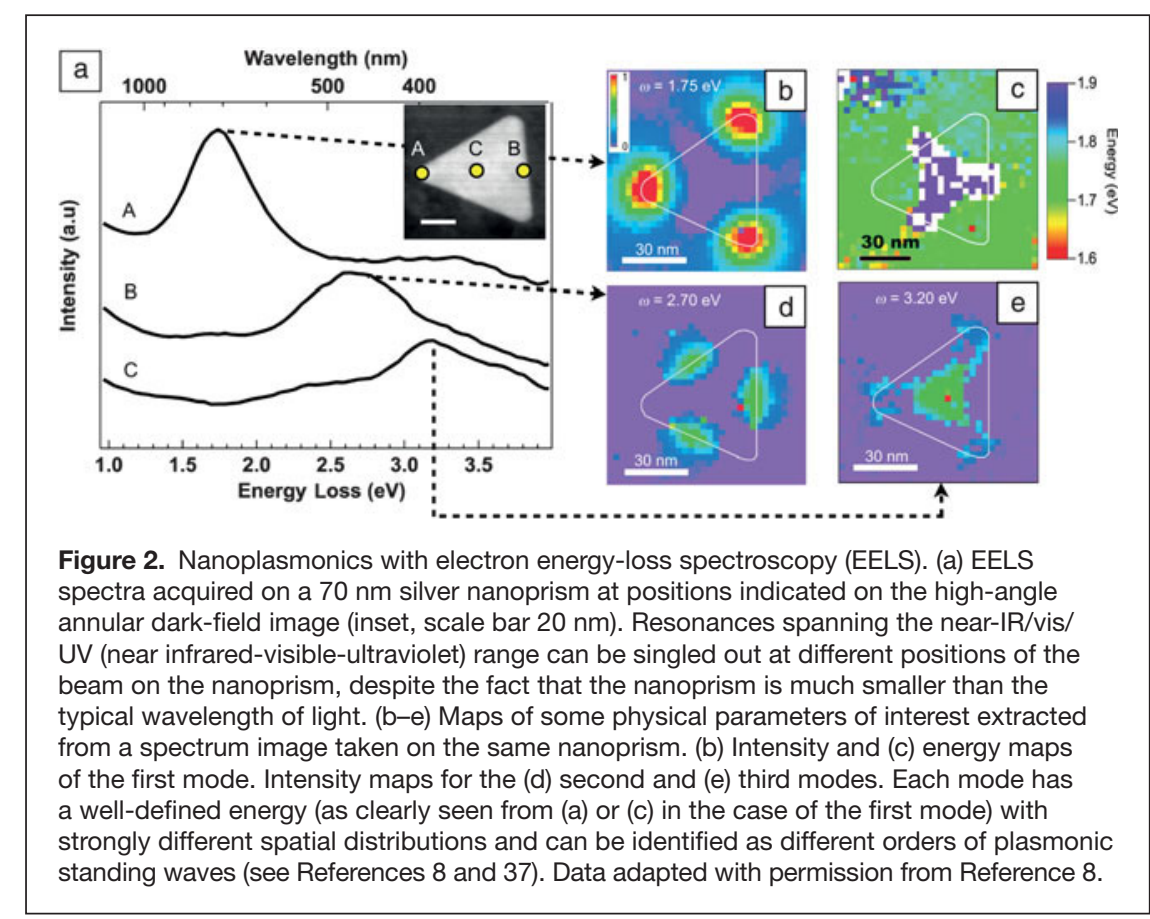




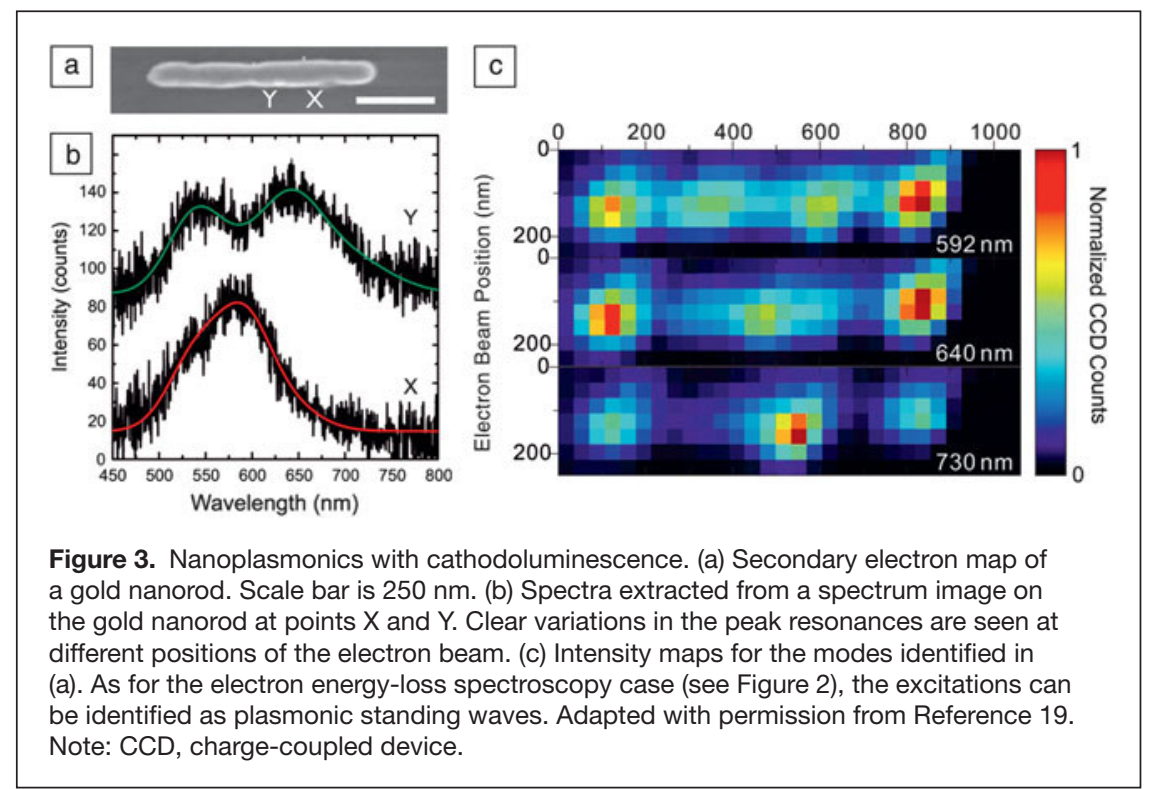

shown to be proportional to polarizabilities ${ }^{20,21}$ or Fresnel coefficients, but the equivalence with optics has been rarely noted until recently. ${ }^{22-24}$ Moreover, although the theory of EELS was strong enough to provide useful simulation tools, such as the boundary element method (BEM) ${ }^{25}$ and the discrete dipole approximation (DDA), ${ }^{26}$ no universal theory linking EELS and optics existed. On the CL front, the pioneering work of Yamamoto not only pointed to the possibility of measuring polarizabilities, but also provided access to the electromagnetic field variation of a simple object (namely mapping the multipole distributions in a metal sphere) for the first time. However, the link between the spatial variations at given energies of EELS or CL was not recognized until recently, ${ }^{23}$ when it was realized that the photonic bandgap crystal eigenmodes could be mapped by EELS. As very similar results could be retrieved either theoretically or experimentally with pure optical spectroscopy, it was a first step toward showing that electron and photon spectroscopies were conceptually similar. The EELS signal was shown to be proportional to the density of electromagnetic states, a central quantity in near-field optics. This idea was extended to arbitrary structures for EELS (and CL in the case of non-absorbing media $)^{27}$ and later adapted to plasmonic structures in the case of CL. ${ }^{28}$ It was thus shown that EELS is very close to the electromagnetic local density of states (EMLDOS) projected along the electron trajectory $(z)$ (zEMLDOS). Such a quantity, already used in the case of scanning near-field optical microscopy, finds a relatively simple interpretation in the case of non-dissipative structures. The case of a dissipative material is more cumbersome, as the very notion of eigenstates is ill-defined. Given the geometry and distribution of materials with various dielectric functions, the Maxwell equations give electrical field eigenfunctions along $z$ for mode $m, E_{z}(\overrightarrow{\mathbf{r}})^{m}$ at position $\mathbf{r}$ and energy $\omega$ and well-defined eigenenergies $\omega_{m}$. The zEMLDOS then yields:

$$
\rho_{z}(\omega, \overrightarrow{\mathbf{r}}) \approx \sum_{m}\left|E_{z}(\overrightarrow{\mathbf{r}})^{m}\right|^{2} \delta\left(\omega-\omega_{m}\right)
$$

The zEMLDOS, and thus in a very good approximation, the EELS (and CL in the case of non-dissipative materials), is thus a map of the $z$-projected eigenfield of energy $\omega_{m}$. There are two points to be noted: First, such a consideration is a radical change of paradigm. Indeed, EELS has been, and still is, mainly used for characterizing material properties of samples of interest, while here, the proposed scheme links EELS to the electromagnetic field properties (as modified by the presence of structured materials). Second, apart from the vectorial form of the EMLDOS, a straightforward analogy can be made between scanning tunneling microscopy and spectroscopy (STM/STS) and STEMEELS. The former aims at mapping eigenstates of the Schrödinger equation, while the latter aims at mapping eigenstates of the Maxwell equation.

In the case of a dissipative material, for which no well-defined eigenenergies exist, the correspondence between the EMLDOS and EELS is strictly established ${ }^{29}$ but needs some adaptation for CL. ${ }^{28}$ However, the eigenmode decomposition (Equation 1) is not well defined, and thus the physical interpretation of the EELS is not easy. Now, as already devised and detailed in the next paragraph, the expanding use of EELS and CL is related to mapping plasmons, excitations that are intrinsically dissipative. Also, experimental facts clearly argue for a description of plasmons in terms of eigenstates. This apparent contradiction can be solved by expressing the zEMLDOS for the field in the vicinity of a metallic particle, the dielectric constant of which is described within the Drude model in the case of small damping. In the Drude model, the dielectric constant is simply dependent on the bulk plasmon energy $\omega_{p}$ and a damping factor $\Gamma$. The surface plasmons then arise at energies $\omega_{m}\left(<\omega_{p}\right)$ and are damped by, essentially, $\Gamma$. In the case of low damping, an expression equivalent to Equation 1 is obtained despite the dissipation, as shown in Equation $2:{ }^{30}$

$$
\begin{gathered}
\rho_{z}(\omega, \overrightarrow{\mathbf{r}}) \approx \\
\frac{2}{\pi} \sum_{m}\left|E_{z}(\overrightarrow{\mathbf{r}})^{m}\right|^{2} \frac{\Gamma \omega_{m}^{2}}{\Gamma^{2} \omega^{2}+\left(\omega^{2}-\omega_{m}^{2}\right)^{2}} \underset{\Gamma \rightarrow 0}{\longrightarrow} \\
\sum_{m}\left|E_{z}(\overrightarrow{\mathbf{r}})^{m}\right|^{2} \delta\left(\omega-\omega_{m}\right)
\end{gathered}
$$

Other innovative schemes using distributions of photon wavenumbers ${ }^{33}$ or polarization ${ }^{34}$ have been used with success but without yet a formal justification.

\section{Applications to the understanding of classical structures: Plasmons and photons}

Knowing that spatially resolved EELS and CL are very good tools for mapping plasmons, what make them relevant over pure optical techniques? The optical techniques now have relatively good spatial resolution but also omnipotent control of polarization and phase that cannot be accessed by EELS, and only the 
polarization can be accessed by CL. Thus, it is not only the gain in spatial resolution that makes EELS/CL experiments much more suitable and the results probably more reliable below $\sim 100 \mathrm{~nm}$, but it is also the possibility of knowing, for all precisely defined energies, the spatial variations of the related excitations and their link to the structure of the sample. In this respect, spectral imaging is a necessity. The first example is from the pioneering work of Yamamoto et al., ${ }^{4}$ who mapped the different multipole spatial distributions at different energies on a silver nanosphere of ca $50 \mathrm{~nm}$ using CL. It allowed for clear establishment of the energy/momentum (dispersion) relation. Such a description can be extended to less symmetric objects such as metallic nanowires, which are the simplest realization of nanoantennas. ${ }^{35}$ From images such as those in Figure 3 (CL surface plasmon polariton mapping on a gold nanowire) and Figure 4 (EELS surface
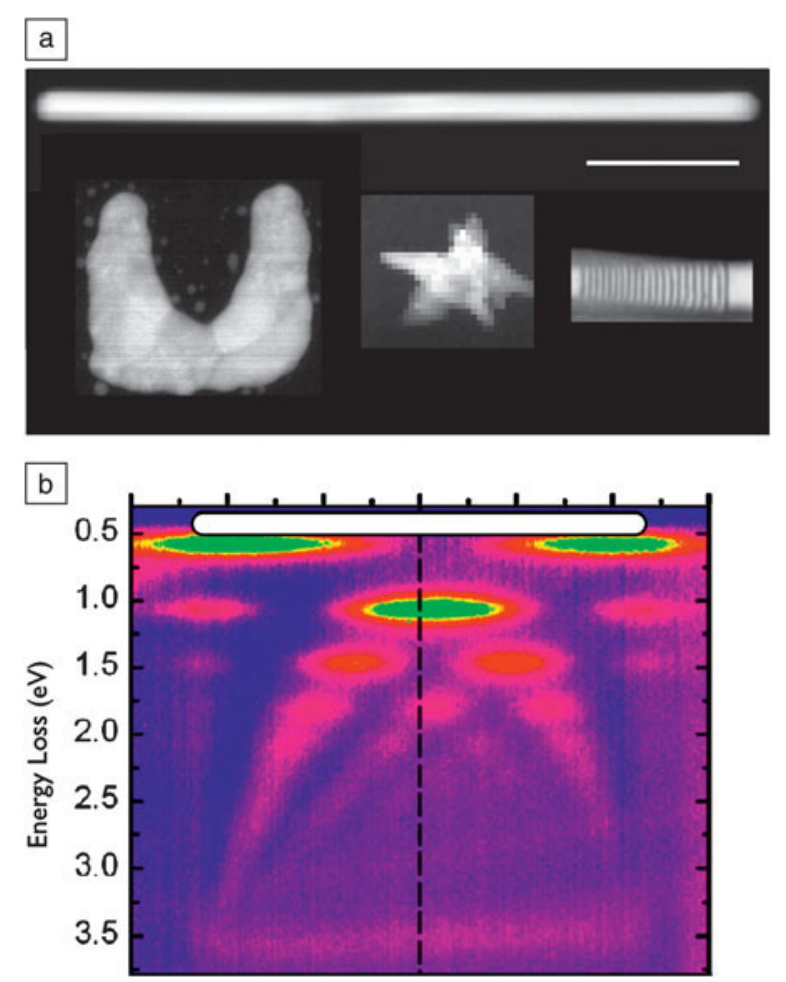

Figure 4. Typical applications of nanoscale electron optical spectroscopies. (a) A montage of different high-angle annular dark-field images of plasmonic nano-objects presented on the same scale. A quantum emitter stack (presented in Figure 7) is also displayed (lower right side) for comparison. The typical resonant wavelength (in vacuum) of the main modes of the structures are around $2200 \mathrm{~nm}$ for the silver nanorod, ${ }^{33} 1200 \mathrm{~nm}$ for the U-shape, lithographied silver split ring resonators (SRRs), ${ }^{39}$ and $890 \mathrm{~nm}$ for the gold nanostar, ${ }^{44}$ much larger than the plasmon wavelength and even greater than the signal localization. Scale bar $=100 \mathrm{~nm}$. (b) Dispersion relation of the plasmonic stationary waves over a straight, chemically grown nanoantenna (silver nanorod). Such a dispersion relation can be taken in a few minutes over the infrared to ultraviolet range, which would be impossible with pure optical spectroscopy. The color scale code ranges from zero electron energy-loss spectroscopy (EELS) intensity (blue) to maximum EELS intensity (green). Adapted with permission from Reference 33. plasmon mapping), which can be established on nano-objects of various sizes (known with typically nanometric precision), dispersion relations can be determined. In these rather symmetric situations, this could also be done (with more difficulty) by optical techniques. ${ }^{36}$ However, the EELS energy range ${ }^{33}$ spans the IR $(\approx 2200 \mu \mathrm{m})$ to $\mathrm{UV}(>200 \mathrm{~nm})$ regime all at once, which is difficult with pure optical means. On less symmetric systems, such as triangular silver nanoplatelets, the dispersion relation, compared to the structure (both edge length and thickness, which can be deduced from EELS ${ }^{15}$ ), can be established and reveals the exact nature of the underlying excitation, namely quasistatic stationary waves, which are fully symmetric in charge distribution with respect to the platelet's horizontal plane of symmetry in the case of thin (typically less than $10 \mathrm{~nm}$ ) triangles. ${ }^{37}$ The excitations in the case of thick $(>70 \mathrm{~nm})$ and large ( $>500 \mathrm{~nm})$ nanoparticles were recently described as being leaky whispering gallery modes ${ }^{34}$ (i.e., surface plasmons traveling on the edges of the nanostructures). More complex resonators can be explored, such as split ring resonators (SRRs) (see a scheme in Figure 5), which are the basic building blocks of many metamaterials. ${ }^{38}$ Optical metamaterials are artificially structured materials with engineered electromagnetic properties that cannot be produced in natural materials. The archetype of such a property is the possibility to produce materials with a negative refractive index.

Such small devices (typically well below half-wavelength dimensions) are intrinsically not emitting when operating in the visible range, and thus are rarely studied by CL. On the other hand, these are artificial materials that are usually fabricated by lithography techniques performed on a thick substrate that cannot be used with EELS. Schemes to adapt lithography to EELS experiments are described in References 39 and 40. Performing spectral imaging in individual SRRs is as important for the understanding of metamaterials as uncovering the energies and spatial distribution of electronic wave functions of individual atoms in real materials. ${ }^{41,42}$ This has been done, as shown in Figure 5. The excitations are stationary plasmonic waves, as confirmed by the simulations. The good agreement between the EELS experimental maps and BEM simulations allows us to consider that the simulations are a good representation of the experiments. Additional information can thus be extracted from the simulations, for example the phase of the excitations, not available from experimental EELS. In these split rings, the excitation spatial distribution is very close to that of a nanorod, but the energy dispersion clearly shows a deviation that can be attributed to the coupling between both legs of the structure. ${ }^{39}$ Finally, more complex distributions of resonators can be studied, in particular the coupling between nanoparticles. ${ }^{40,43}$ One open question in coupled nanoparticles is the strength of the field in the gap between two particles, for the so-called bright mode - a dipolar mode that easily couples to free space light, per opposition to quadrupolar modes that hardly couple to light. As this mode couples to light, it has primarily an electrical eigenmode that is polarized along the direction linking the two particles, which, in practice, is perpendicular to the electron 
beam path $(z)$. As the EELS and CL are close to the zEMLDOS, they will display zero intensity in the gap of the dimer and cannot be used efficiently for such studies.

Spectral imaging EELS is also very well adapted to the study of plasmons at the scale of a few nanometers. Figure 6 shows an example of the energy position and intensity distribution of the EELS signal over a $100 \mathrm{~nm}$ diameter gold nanostar. It is striking to note that the plasmons not only peak on the tips, but their energies depend on which tip they are located on. This means that they are localized on a very short scale, much smaller than the wavelength and even much smaller than the whole metallic structure. This is in great contrast to the previous examples where, although the intensity peaked obviously at maxima of the oscillations, the excitations were delocalized over the entire object size - several hundreds of nanometers. This high spatial localization, analyzed in detail in Reference 44 , can be attributed to the loss of spatial coherence between different tips linked together by a core larger than the optical skin depth (i.e., the typical distance over which the electromagnetic field is screened in a metal), thus impeding cross-talk between oscillations of two tips. Such effects, impossible to directly study with full optical methods, ${ }^{45}$ have implications on actual surfaceenhanced Raman spectroscopy activity of these nanostars. ${ }^{46}$

Finally, although the spatial resolution needed is much less stringent than the one already shown for plasmons, it is worth emphasizing the relevance of using $\mathrm{CL}^{47}$ and EELS ${ }^{23,48}$ for the analysis of photonic and plasmonic bandgap crystals and their defects.

\section{Mapping of the emission of quantum emitters}

Figure 5. (a) Experimental intensity maps for the two first modes of a lithographed silver split ring resonator. The $\mathrm{A} 1$ mode is the first charge antisymmetric mode, and $\mathrm{S} 1$ is the first charge symmetric one. Scale bar is $50 \mathrm{~nm}$. (b) Boundary element method simulated electron energy-loss spectroscopy (EELS) maps of the same modes. Experimental spatial variations are well reproduced by the simulations. The corresponding simulated eigencharges' (c) amplitude and (d) phase are displayed. Color scale for the amplitude is zero for blue and maximum for red; the phase $\pi$ (negative) is blue and 0 (positive) for red. These clearly reveal the charge stationary wave nature of the modes. Adapted with permission from Reference 39.

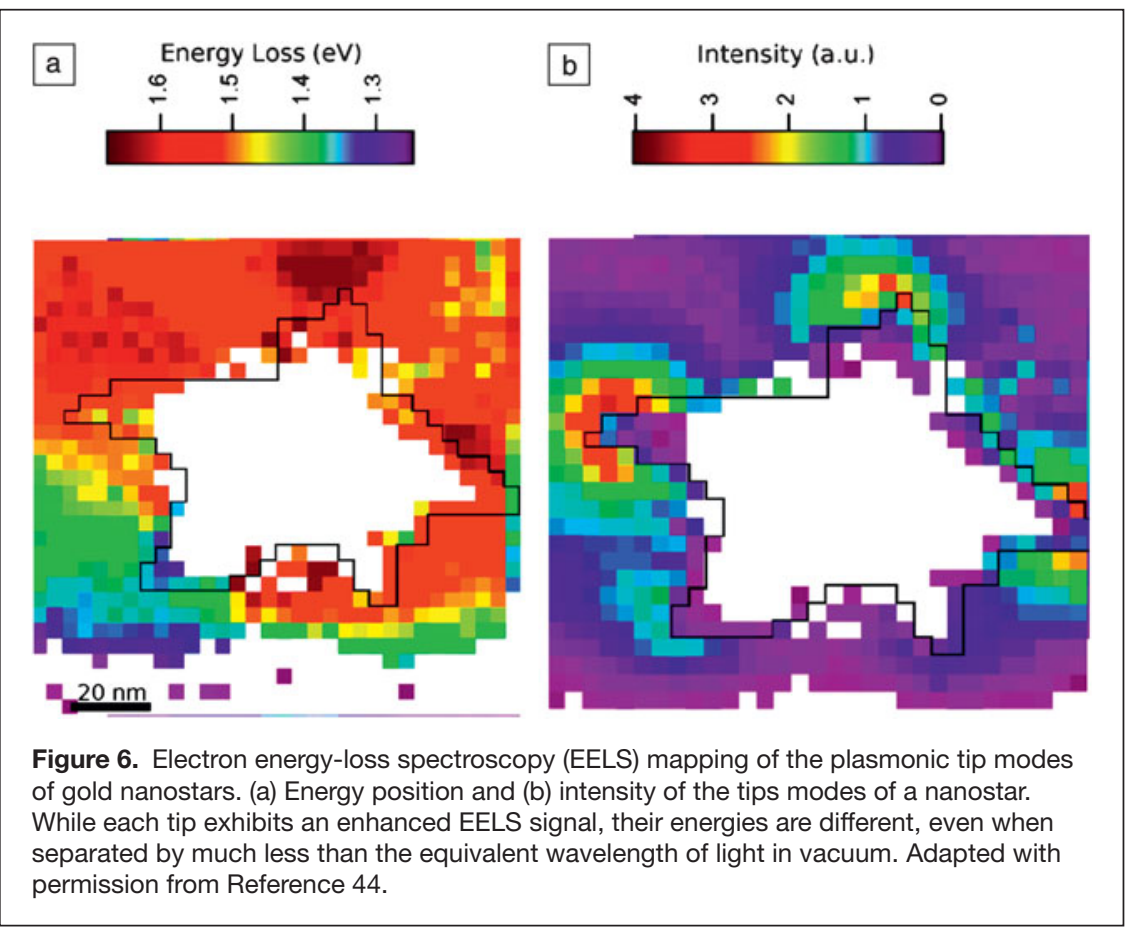

Up to here, the problems considered have involved changes in optical properties of nanoparticles linked to a change in boundary conditions of the Maxwell equations. However, for various applications (LEDs, lasers, photodetectors), engineering the optical properties of semiconductors is commonly done by taking advantage of quantum confinement (namely changes in the Schrödinger equation boundary conditions). Studying such systems requires the knowledge of the structural properties down to the single atomic layer and of their optical properties down to the nanometer scale. In this regard, EELS combined with high-resolution imaging could be considered a natural solution. However, EELS suffers from genuine limitations: the energy resolution is limited, both because of technical issues (nonmonochromaticity of the electron gun) and fundamental ones. It is known that the absorption signals such as EELS is larger than the related luminescence, and the size of the delocalized signal ${ }^{49}$ is usually much larger than the size of individual nanostructures. ${ }^{50}$ On the other hand, CL has a longstanding track record of mapping semiconductor emissions in bulk materials. ${ }^{6}$ It has been recently adapted to smaller structures and in particular with some success to semiconducting nanowires, ${ }^{7}$ but without the relevant spatial resolution to access individual quantum confined structures. However, the application 
of CL at small scales has been limited by the weak collection efficiency of the commercial CL instrument and by the belief that the photon generation mechanism was highly delocalized in nature. This mechanism is often caused by charge carrier diffusion: electron-hole pairs at all energies are generated at the electron beam impact position. They migrate over their diffusion length and may recombine at various places with an emission energy related to the properties of the material at the recombination position rather than at the position of the electron beam.

Recently, using a high-throughput, dedicated STEM CL system, Zagonel et al. ${ }^{10}$ showed the possibility of singling out the emission wavelength of individual quantum discs (QDs) of nanometer sizes and spaced a few nanometers apart. They also showed the possibility of correlating this optical information to the size of the QDs measured with monolayer precision, opening the possibility to characterize quantum confined systems at their relevant length.

Figure 7 presents the output of a CL spectral imaging experiment on a stack of GaN/ AlN cylindrical quantum discs embedded in a GaN/AlN nanowire. Spectra clearly display well-defined peaks appearing at different wavelengths for different electron beam positions. As a full spectrum with sometimes several peaks is taken at each pixel (typically $1 \mathrm{~nm}$ step size), the information is rather rich, and a first possibility is to map the wavelength position, the intensity, and the full width at half maximum of the most intense peak in each spectrum (Figure 8). The wavelength position map displays clear areas of constant values, while the intensity map clearly shows variations along and perpendicular to the wire direction. Some of this information can be directly related to an individual quantum well position (white contrast in the HAADF). A step further in the analysis can be done by using the full spectral-spatial information contained in a spectrum image ${ }^{12}$ and is discussed in detail in Reference 10. The main output is that the emission wavelength of any quantum disc, as small as four monolayers, can be singled out even if the next one is less than $4 \mathrm{~nm}$ away. Here, it is worth noting that charge carrier diffusion, which usually broadens the CL spatial resolution in a bulk material, is severely reduced in quantum confined heterostructures and has been proven not to limit the spatial resolution in the present case. ${ }^{10}$

Comparison with their actual thickness measured in monolayers (see Figure $8 \mathrm{~b}$ ) showed dispersion with the size, as expected for quantum confinement. In addition, an effect of the local environment (pressure, local doping) can be seen. Indeed, for the same thickness and same environment, the theory predicts the same emission wavelength, which is not the case for different QDs with the same thickness yet different position along the nanowire, and thus different direct environment. It is worth noting that such measurements, made at the relevant scale, challenge current state-of-the-art simulation techniques because such complex structures are difficult to simulate.

\section{Conclusion and outlook}

This article has shown how powerful electron-based optical techniques and simulation tools can be used in unraveling the physics and optical properties of nano-objects, should they be classical or quantum in nature. In comparing both techniques, it is worth noting that by definition, all inelastic effects are
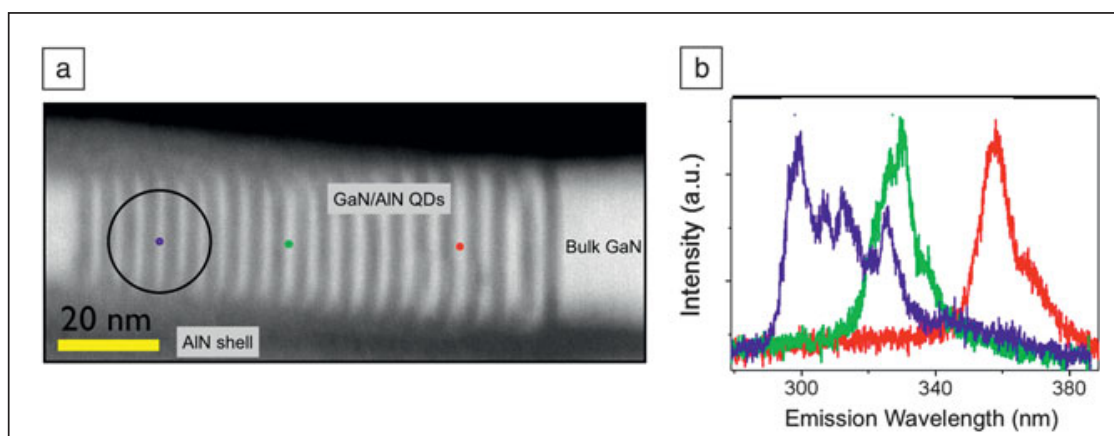

Figure 7. Cathodoluminescence spectral imaging of a stack of individual quantum emitters made up of GaN in AIN quantum discs (QDs) in a scanning transmission electron microscope. (a) High-angle annular dark field (HAADF) of a typical nanowire with embedded QDs. GaN appears brighter, AIN darker. The circle shows the best spatial resolution reported in a scanning electron microscope, ${ }^{7}$ and the small colored spots show the typical probe size in the presented results. (b) Spectra extracted at positions noted in (a) from spectrum images. Adapted with permission from Reference 10.
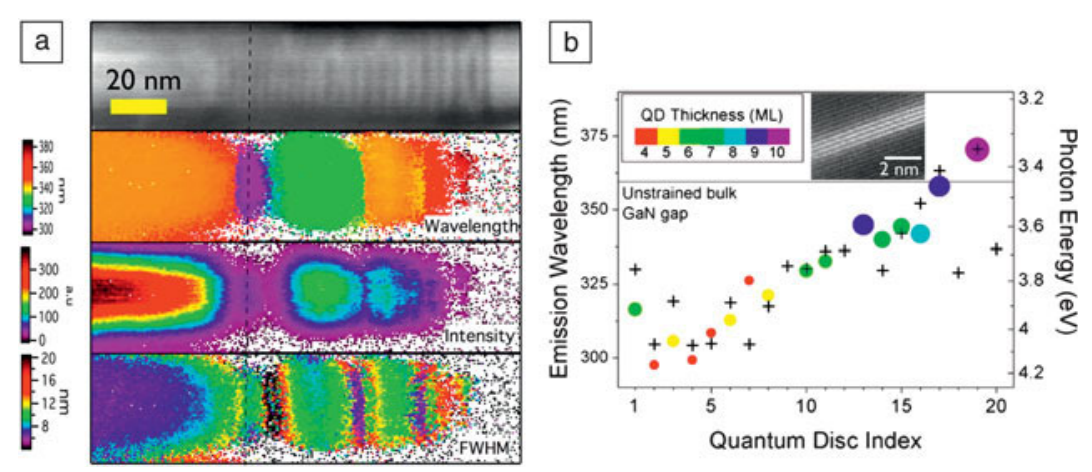

Figure 8. (a) Two-dimensional map extracted from the spectrum image of the principal emission peaks in a quantum emitter structure, featuring (from top to bottom) the highangle annular dark field (HADDF) image, wavelength position, intensity, and full width at half maximum (FWHM) of the most intense peak. A direct correlation between the structure (HAADF) and some emission properties can be seen for some discs (e.g., the dashed line). (b) Using a systematic analysis of all data in the spectrum image (see Reference 10 for details), every emission line can be singled out and attributed to a given quantum disc (QD) indexed from its position with respect to the left of the image. The QD emission wavelength can then be compared to its size, measured in monolayers (see inset). In addition, the emission wavelength dependence on the position of the QD, given by its index and thus its position along the nanowire, can be evaluated. 
reflected in energy loss events. Almost all electrons that have been inelastically scattered can be detected by electron energyloss spectroscopy (EELS), the typical angle of inelastic scattering (tens or hundreds of microradians) being much smaller than the detection angle. Nowadays, with the availability of very high sensitivity EELS detection systems, this means that detecting an EELS signal is a very efficient process. In contrast, cathodoluminescence $(\mathrm{CL})$ is a second-order process, and if the quantum efficiency is weak, the CL signal will also be weak. Moreover, the collection efficiency is usually very poor, because the space available to place a high numerical aperture collection system is small between the specimen and the objective lens pole piece. In the case of signals that have similar spectral profiles in EELS and CL, and for which the emission quantum efficiency is weak-typically plasmonic signals in small nanoparticles-EELS is always the technique of choice if energy resolution or access to the polarization is not of concern. On the other hand, for signals with sharp peaks and high quantum efficiency systems, for which energy resolution is relevant, CL may take the lead.

These techniques, which have been recently adapted to the study of standard samples for plasmonic, photonic, or quantum systems, will certainly play a decisive role in the understanding of new nano-optical devices. It should also be noted that the next generation of scanning transmission electron microscopes, encompassing both monochromators and aberration correctors, will allow us to obtain EELS spectra with very small influence of the zero loss signal (which usually obscures the signal at low energy loss), high spectral resolution, and still a high signal-tonoise ratio. ${ }^{33}$ This is already popularizing the use of EELS for nano-optics.

However, the experiments described here are limited to linear and classical nano-optics. A few proposals ${ }^{27}$ and the experimental realization of part of one of them ${ }^{51}$ benefit from the combination of the spatial resolution of the electron beam and the high spectral resolution and the high intensity provided by a laser. Combining both of them clearly paves the way to another exciting field: non-linear and quantum nano-optics with fast electrons.

\section{Acknowledgments}

M.K. acknowledges all of the STEM group collaborators and students participating in the emergence of this topic in the group over the years, and in particular O. Stéphan, C. Colliex, L. Zagonel, J. Nelayah, S. Mazzucco, G. Boudarham, A. Gloter, and M. Tencé. He acknowledges useful collaboration and discussions with L. Henrard and coworkers, L. Liz-Marzan and coworkers over the years, and more recently the teams of M. Tchernycheva and M. Wegener. F.J.G.A is thankful to the groups of Orsay (C. Colliex), Vigo (L. Liz-Marzan), AMOLF (A. Polman), and Southampton (N. Zheludev) for many enjoyable interactions over the last years. W. Sigle, G. Botton, and the team of A. Polman are gratefully acknowledged for giving access to preprints and/or giving the right to reproduce their work. The authors acknowledge financial support from the European
Union under the Framework 6 program under a contract for an Integrated Infrastructure Initiative. Reference 026019 ESTEEM, and French ANR (1010 BLAN 1016 "HYNNA") support.

\section{References}

1. P.E. Batson, Phys. Rev. Lett. 49, 936 (1982)

2. F. Ouyang, P.E. Batson, M. Isaacson, Phys. Rev. B 46, 15421 (1992)

3. I.R. Khan, D. Cunningham, S. Lazar, D. Graham, W.E. Smith, D.W. McComb, Faraday Discuss. 132, 171 (2006).

4. N. Yamamoto, K. Araya, F.J.G. de Abajo, Phys. Rev. B 64, 205419 (2001)

5. S.J. Pennycock, A. Howie, Philos. Mag. A 41, 809 (1979).

6. H.P. Strunk, M. Albrecht, H. Scheel, J. Microsc. (Oxford) 224, 79 (2006).

7. U. Jahn, J. Ristic, E. Calleja, Appl. Phys. Lett. 90, 161117 (2007).

8. J. Nelayah, O. Stéphan, M. Kociak, F.J. Garcia de Abajo, L. Henrard, I. PastorizaSantos, L.M. Liz-Marzan, C. Colliex, Microsc. Microanal. 13, 144 (2007).

9. M. Bosman, V.J. Keast, M. Watanabe, A.I. Maaroof, M.B. Cortie, Nanotechnology 18, 165505 (2007)

10. L.F. Zagonel, S. Mazzucco, M. Tencé, K. March, R. Bernard, B. Laslier, G. Jacopin M. Tchernycheva, L. Rigutti, F.H. Julien, R. Somguang, M. Kociak, Nano Lett. 11, 568 (2011).

11. F.J. Garcia de Abajo, Rev. Mod. Phys. 82, 209 (2010).

12. M. Kociak, O. Stéphan, M.G. Walls, C. Colliex, Spatially Resolved EELS

The Spectrum-Imaging Technique and Its Applications (Springer, NY, 2011),

C. 4 , p. 163 .

13. C. Colliex, J. Electron Microsc. 60, S161 (2011).

14. C. Jeanguillaume, C. Colliex, Ultramicroscopy 28, 252 (1989).

15. R. Egerton, Electron Energy Loss Spectroscopy in the Electron Microscope (Plenum, New York, 1986).

16. J. Nelayah, J. Gu, W. Sigle, C.T. Koch, I. Pastoriza-Santos, L.M. Liz-Marzan, P.A. van Aken, Opt. Lett. 34, 1003 (2009).

17. B. Schaffer, W. Grogger, G. Kothleitner, F. Hofer, Ultramicroscopy 110, 1087 (2010).

18. B. Schaffer, G. Kothleitner, W. Grogger, Ultramicroscopy 106, 1129 (2006).

19. E.J.R. Vesseur, R. de Waele, M. Kuttge, A. Polman, Nano Lett. 7, 2843 (2007).

20. T.L. Ferrell, P.M. Echenique, Phys. Rev. Lett. 55, 1526 (1985).

21. M. Kociak, O. Stéphan, L. Henrard, V. Charbois, A. Rothschild, R. Tenne, C. Colliex, Phys. Rev. Lett. 8707, 075501 (2001).

22. D. Taverna, M. Kociak, V. Charbois, L. Henrard, O. Stéphan, C. Colliex, J. Electron. Spectrosc. Relat. Phenom. 129, 293 (2003).

23. F.J.G. de Abajo, A.G. Pattantyus-Abraham, N. Zabala, A. Rivacoba, M.O. Wolf, P.M. Echenique, Phys. Rev. Lett. 91, 143902 (2003).

24. R. Arenal, O. Stéphan, M. Kociak, D. Taverna, A. Loiseau, C. Colliex, Phys. Rev. Lett. 95, 127601 (2005).

25. F.J. García de Abajo, J. Aizpurua, Phys. Rev. B 56, 15873 (1997).

26. L. Henrard, P. Lambin, J. Phys. B: At. Mol. Opt. Phys. 29, 5127 (1996).

27. F.J. Garcia de Abajo, M. Kociak, New J. Phys. 10, 073035 (2008).

28. M. Kuttge, E.J.R. Vesseur, A. Polman, Appl. Phys. Lett. 94, 183104 (2009).

29. F.J. Garcia de Abajo, M. Kociak, Phys. Rev. Lett. 100, 106804 (2008).

30. G. Boudarham, PhD thesis, Université Pierre et Marie Curie-Paris VI (2011).

31. F.J. Garcia de Abajo, A. Howie, Phys. Rev. Lett. 80, 5180 (1998).

32. N. Geuquet, L. Henrard, Ultramicroscopy 110, 1075 (2010).

33. D. Rossouw, M. Couillard, J. Vickery, E. Kumacheva, G.A. Botton, Nano Lett. 11, 1499 (2011)

34. L. Gu, W. Sigle, C.T. Koch, B. Ögüt, P.A. van Aken, N. Talebi, R. Vogelgesang, J.L. Mu, X.G. Wen, J. Mao, Phys. Rev. B 83, 195433 (2011).

35. L. Novotny, N. van Hulst, Nat. Photonics 5, 83 (2011).

36. J. Dorfmüller, R. Vogelgesang, R. Weitz, C. Rockstuhl, C. Etrich, T. Pertsch, F. Lederer, K. Kern, Nano Lett. 9, 2372 (2009).

37. J. Nelayah, M. Kociak, O. Stéphan, N. Geuquet, L. Henrard, F.J.G. de Abajo, I. Pastoriza-Santos, L.M. Liz-Marzan, C. Colliex, Nano Lett. 10, 902 (2010).

38. G. Dolling, M. Wegener, C.M. Soukoulis, S. Linden, Opt. Lett. 32, 53 (2007) 39. G. Boudarham, N. Feth, V. Myroshnychenko, S. Linden, J. García de Abajo, M. Wegener, M. Kociak, Phys. Rev. Lett. 105, 255501 (2010).

40. A.L. Koh, A.I. Fernandez-Dominguez, D.W. McComb, S.A. Maier, J.K.W. Yang, Nano Lett. 11, 1323 (2011)

41. J. Zuo, M. Kim, M. O’Keeffe, J. Spence, Nature 401, 49 (1999).

42. S.G. Lemay, J.W. Janssen, M. van den Hout, M. Mooij, M.J. Bronikowski, P.A. Willis, R.E. Smalley, L.P. Kouwenhoven, C. Dekker, Nature 412, 617 (2001)

43. M. Chu, V. Myroshnychenko, C. Chen, J. Deng, C. Mou, F. Garcia de Abajo, Nano Lett. 9, 399 (2009). 
44. S. Mazzucco, 0. Stéphan, C. Colliex, I. Pastoriza-Santos, L.M. Liz-Marzan, F.J. Garcia de Abajo, M. Kociak, Eur. Phys. J. Appl. Phys. 54, 33512 (2011). 45. F. Hao, C.L. Nehl, J.H. Hafner, P. Nordlander, Nano Lett. 7,729 (2007). 46. L. Rodriguez-Lorenzo, R.A. Alvarez-Puebla, I. Pastoriza-Santos, S. Mazzucco, 0. Stéphan, M. Kociak, L.M. Liz-Marzan, F.J.G. de Abajo, J. Am. Chem. Soc. 131, 4616 (2009).

47. K. Takeuchi, N. Yamamoto, Opt. Express 19, 12365 (2011).
48. J. Cha, Z. Yu, E. Smith, M. Couillard, S. Fan, D. Muller, Phys. Rev. B 81, $113102(2010)$

49. L. Gu, W. Sigle, C.T. Koch, J. Nelayah, V. Srot, P.A. van Aken, Ultramicroscopy 109, 1164 (2009).

50. M. Couillard, M. Kociak, O. Stéphan, G.A. Botton, C. Colliex, Phys. Rev. B 76, 165131 (2007)

51. B. Barwick, D.J. Flannigan, A.H. Zewail, Nature 462, 902 (2009).

\section{《e NDNC 2012 New Diamond and Nano Carbons Conference \\ May 20-24, 2012 Conrad San Juan Condado Plaza - San Juan, Puerto Rico}

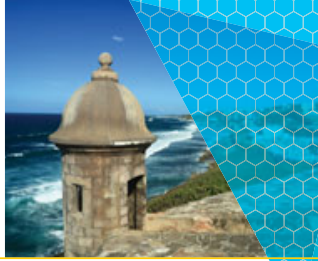

\section{SCIENTIFIC PROGRAM}

The five-day conference will feature oral and poster presentations covering:

- Science and Technology of Diamond

- Synthesis and Characterization of Graphenes

- Synthesis and Characterization of Nano Carbons

- C-based Coatings and Tribological Applications

- Interfaces and Heterojunctions of C-based Materials with Other Materials

- Electronic, Optical and Acoustic Applications of C-based Materials
- Electrochemical Applications of C-based Materials

- Sensor Applications of C-based Materials

- Biological Applications of C-based Materials

- Spintronics of C-based Materials

- Business Opportunities and Commercialization

www.mrs.org/ndnc-2012
IMPORTANT DATES

Abstract Deadline February 15, 2012

Pre-registration Opens February 21, 2012

Pre-registration Ends May 3, 2012

\section{DECONVEELS}

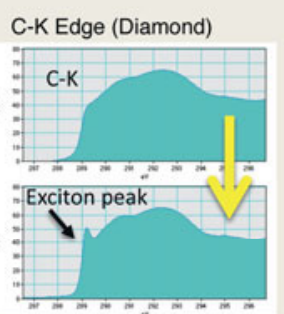

Raw data: Courtesy of Dr. P. Batson
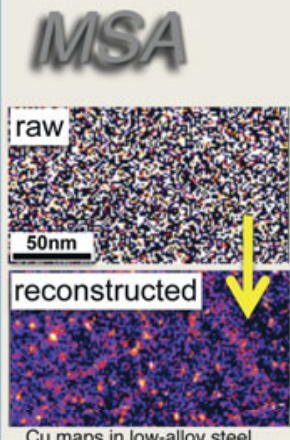

\section{Electron Energy Loss} Spectrum Deconvolution

Software Monochromator for EELS

DeConvEELS rectifies an Electron Energy Loss Spectrum (EELS) by deconvoluting with a low-loss or zero-loss spectrum

\section{Key Features}

- Advanced Deconvolution Algorithms: DeConvEELS uses Maximum Entropy Method or Richardson-Lucy Algorithm - Easy-to-use User Interface: DeConvEELS normally works with default setups, while the user can change its setups easily within a custom-made setup dialog.

- SI Module (Option): EELS SI (Spectrum Image) data can be handled easily using this module.

\section{Multivariate Statistical Analysis}

Advanced Manipulation Tools for Spectrum Images (SIs)

MSA finds statistically significant features from $2 D$ and $3 D$ spectrum images gathered by XEDS, EELS, EFTEM, CL, etc.

\section{Key Features}

- Automatically extracts statistically significant spectral features and corresponding spatial amplitudes as principal components

- Performs efficient noise reduction on SIs

- Enhances weak signals hidden under noise in SIs

- Includes utilities to import line profiles and SIs obtained by other acquisition systems than Gatan DigitalMicrograph.

\section{Details can be found at:} www.hremresearch.com

\section{FFT-Multislice Simulation Suite} XHREM (WinHREM/MacHREM) STEM Extension (32/64 bit)/Cluster CBED Extension (32/64 bit)

\section{DigitalMicrograph Plug-ins}

HREM-Filters Pro/Lite

FTSR (Focal and Tilt Series Reconstruction) IWFR (Iterative Wave Function Reconstruction)

DeConvEELS (Deconvolution for EELS)

DeConvHAADF (Deconvolution for STEM-HAADF)

MSA (Multivariate Statistical Analysis)

QPt (Quantitative Phase Imaging Technology)

GPA (Geometrical Phase Analysis)

PPA (Peak Pairs Analysis)

New HoloDark (DarkField Holography for Strain)

New QED (Quantitative Electron Diffraction)

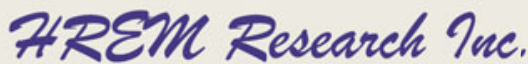

Higashimatsuyama, Saitama JAPAN support@hremresearch.com 